\title{
Reliability of the Probability Effect on Event-related Potentials during Repeated Testing
}

\author{
SAYAKA KINOSHITA, HISAO MAEDA, JUN NAKAMURA, \\ EISUKE KODAMA AND KIICHIRO MORITA \\ Department of Neuropsychiatry, Kurume University \\ School of Medicine, Kurume 830, Japan
}

Received for publication August 23, 1995

\begin{abstract}
Summary: The reliability of event-related potentials (ERPs) was studied in 10 healthy adults who were tested 8 times over 7-10 day intervals using a standard auditory oddball paradigm. The difference waveforms, obtained by subtracting the averaged waveforms for frequent trials from those obtained in rare trials, were designed to analyze the components of the ERPs, such as the P300, and to focus on the reliability of the probability effect on the ERPs. The between-session reliability ( 8 sessions) and the within-session reliability (order of blocks or of different visual procedures) were computed for the obtained difference waveforms. The between-session reliabilities, expressed as the intraclass correlations ( $r^{\prime}$ ) for the P300 amplitude, area and latency, were $0.70,0.61$ and 0.65 , respectively. The within-session reliability, presented as the Pearson correlation coefficients (r) for the three P300 measures were $0.43,0.35$ and 0.25 for different eyes. The values were $0.45,0.39,0.42$ between the first and the second blocks (eyes-open) and 0.58, 0.47 and 0.29 (eyes-closed). These findings indicate that the P300 amplitude calculated from the difference waveforms may be the most stable marker for the between-session reliabilities. There were no significant differences in the P300 measures over the 8 sessions, suggesting that habituation may not occur with the difference waveform reflecting the probability effect on ERPs. The difference waveform may be useful in research on repetitive group ERPs.
\end{abstract}

Key words: reliability - event-related potential — probability effect difference waveform - habituation

\section{Introduction}

Event-related potentials (ERPs) have been evaluated as markers to investigate the mechanisms of information processing in healthy subjects and in patients with cognitive disorders such as schizophrenia, depression, or dementia (Pritchard,1986, 1987; Blackwood et al. 1987; Polich, 1991). While information on the reliability or agreement of ERP measures is important, few such studies have been performed on P300 parameters (see Table 1 of Segalowitz and Barnes, 1993).

Correspondence and reprint requests to: Sayaka Kinoshita, M.D., Department of Neuropsychiatry, Kurume University School of Medicine, 67 Asahi-machi, Kurume 830, Japan. 
Several oddball tasks were used in a reliability study (see Table 1 of Segalowitz and Barnes, 1993). Most research, however, has evaluated variations in the P300 amplitude and latency. Using the auditory oddball paradigm, four studies have reported a within-session reliability (Pearson correlation coefficient) for the P300 latency ranging from 0.32 to 0.84 (Sklare and Lynn, 1984; Polich, 1986; Fabiani et al. 1987; Segalowitz and Barnes, 1993). Three studies report the reliability of the P300 amplitude to be $0.48,0.65$, and 0.93 (Polich, 1986; Fabiani et al. 1987; Segalowitz and Barnes,1993). In studies of between-session reliability, the Pearson's $r$ values were 0.32 to 0.93 for the P300 latency and 0.62 to 0.93 for the P300 amplitude. Segalowitz and Barnes (1993) proposed that the intraclass correlation (r') (Shrout and Fleiss, 1979) was appropriate for statistical analysis of reliability, and reported details of the between- and within-session reliabilities for the N100, P200 and P300 components of the ERP. The intraclass correlation coefficients for the latency of these components ranged from 0.27 to 0.71 , and for the amplitude from 0.09 to 0.62 . The authors found that the P300 had significant stability in terms of both state (withinsession reliability) and trait (betweensession reliability). However, no studies have evaluated the reliability of the P300 when measuring it more than three times. Only one retest was carried out to examine reproducibility. It is important to determine whether the variability of the ERP is due to a state or trait effect, especially in clinical applications. Thus reliability should be evaluated in repeated sessions at specified intervals to search for stable parameters.
The probability effect is one of the most common influences on the ERPs (Fabiani et al. 1987; Polich, 1991). The reproducibility of the probability effect on the ERP should be evaluated to determine the mechanism of ERP generation.

The present study was intended to evaluate the reliability of the probability effects on the N100, N200 and P300 measures (amplitude, area and latency) estimated within one experimental session (within-session reliability) and across 8 sessions (between-session reliability) at intervals of 7-10 days, except for one session performed after one month between sessions 6 and 7, by using the rare waveform minus the frequent waveform. Reliable clinical tools for use as markers of state or trait are also discussed.

\section{Materials and Methods}

\section{Subjects}

Ten Japanese subjects (men) between the ages of 29 and 52 years (mean $=38$ years) were evaluated. All were righthanded, as assessed by the Raczkowski and Kalat (1974) inventory. All were healthy, had normal hearing, had no history of psychiatric disorders or of drug or alcohol dependencies. Each gave their informed consent for participation.

\section{Recording procedures}

Each session consisted of a series of 6 blocks with a rest interval of $5 \mathrm{~min}$. Subjects were required to complete 4 blocks: two blocks were done with the eyes open (open in first and second blocks) or eyes closed (closed in first and second blocks). Task-free recordings were performed after each eye procedure with 
the eyes open or closed. The order of blocks was counterbalanced across the sessions for each subject. In the eyes-open procedure, the subjects were asked to limit their gaze to a square $(7 \times 7 \mathrm{~cm})$ located $80 \mathrm{~cm}$ from the floor and $150 \mathrm{~cm}$ in front of the subject's chair, so as to minimize ocular movements (visual fixed procedure): Tones were administered binaurally through headphones. All subjects were presented with a test tone for 5 min before the first recording.

Each subject completed a total of 8 sessions at intervals of 7-10 days between sessions 1 through 6 , and between sessions 7 and 8. However, there was a one-month interval between sessions 6 and 7 .

\section{Recording apparatus}

Recordings were obtained between 2 and 4 PM with the subject seated in an armchair in a sound-proof, electrically shielded room. ERPs were elicited by two tones, one high pitched (2000 Hz: rare) and one low pitched (1000 Hz: frequent) at $70 \mathrm{~dB}$ SPL, having a $10 \mathrm{~ms}$ rise-fall and $100 \mathrm{~ms}$ plateau times. The frequent tone occurred $80 \%$ of the time, while the rare tone occurred $20 \%$ of the time. Tones were presented in a random series at a mean rate of $0.7 \mathrm{~Hz}$. The subject's task was to respond by pressing a button with the right hand to the high tone (rare) and count the number of the high tone responded.

The electroencephalogram (EEG) was recorded at $\mathrm{Fz}$ and $\mathrm{Cz}$ electrode sites of the 10-20 system, referred to the linked mastoids. $\mathrm{Ag} / \mathrm{AgCl}$ electrodes were used for each placement. The gland electrode was placed on the subject's forehead. Impedance for all recording sites was consistently below $5 \mathrm{~K} \Omega$. The low filter bandpass was 0.5 and the high filter bandpass was $50 \mathrm{~Hz}$. The waveforms were amplified and averaged on-line using a Nihon-Kohden MEB 5304 (Tokyo, Japan). The recording epoch began $100 \mathrm{~ms}$ before the onset of the stimulus and lasted 1000 ms. The baseline was estimated by computing the average activity in the $100 \mathrm{~ms}$ period preceding the stimulus.

The ERP data reported were difference waveforms that were obtained by subtracting the waveforms elicited by the frequent stimuli from the averaged waveforms evoked by the rare stimuli at the $\mathrm{Cz}$ recording site. The subtracted waveforms have been defined as a reflection of the probability effect (Fabiani et al. 1987). The $\mathrm{Cz}$ site has been recommended for the analysis of multiple ERP components such as N100, N200 and P300 (Polich, 1991; Segalowitz and Barnes, 1993). In all blocks, 20 target artifact-free waveforms were used for subsequent analysis. Artifact rejection was set by an acceptance window of $\pm 50 \mu \mathrm{V}$ at the $\mathrm{Fz}$ and $\mathrm{Cz}$.

\section{Definition and measurement of ERPs components}

Component latencies were defined and scored manually as follows: N100, the most negative peak between 50 and 130 ms; N200, the most negative peak between 160 to 300 ms before P300; and P300, the largest positive peak between 250 and $500 \mathrm{~ms}$. The averaged waveform was filtered at $24 \mathrm{~Hz}(-3 \mathrm{~dB})$ and used for measurement. Peak-picking methods for the ERP' measurements (Fabiani et al. 1987) were used. The P300 area was measured by integration of the positive values between the same time windows (250-500 ms). 


\section{Statistical analysis}

One factor repeated ANOVA was used to compute the difference between sessions with a checking epsilon (G-G, H-G epsilon). For ERP measures with statistically significant differences between sessions (epsilon <1.0), the one-factor ANOVA was used to examine the main session effect for the group. Fisher's protected LSD was then applied as the post-hoc test across 8 sessions. Next, a two-factor ANOVA (session $\times$ subject, session $\times$ condition, session $\times$ order ) was conducted to evaluate interactions. A twofactor ANOVA (session $\times$ subject for two visual procedures, with eyes-open and eyes-closed conditions) was performed for intraclass correlation (see below). The between-session reliability was expressed as the intraclass correlation coefficient $\left(\mathrm{r}^{\prime}\right)$ as reported by Shrout and Fleiss (1979). The within-session reliability was calculated using Pearson's productmoment correlation coefficient (r). Bartlett's T-test was used to evaluate statistical significance. A level of $\mathrm{P}<0.05$ was accepted as statistically significant. In the present paper, a value over 0.5 was considered a generally acceptable value (Helmstadter, 1964).

\section{Results}

\section{ERPs of difference waveforms}

Between-session reliability $\left(r^{\prime}\right)$ : intraclass correlation : For the P300 measures with both the eyes-open and eyes-closed procedures, the intraclass correlation coefficients (the between-session reliability) for the P300 base-to-peak amplitude (P300 amplitude), area and latency were $0.70,0.61$ and 0.65 , respectively. The correlation coefficients for the N100-P300, N200-P300 peak-to-peak amplitude (N100P300, N200-P300 amplitude) were 0.61 and 0.43 , respectively. The betweensession reliability for the N100, N200 base-to-peak amplitude (N100, N200 amplitude) and latency were $0.18,0.48$ and $0.05,0.30$, respectively. The values of the intraclass correlation coefficients for the amplitudes of the N100, N200 and

TABLE 1.

Between-session reliability $\left(r^{\prime}\right)$ (Intraclass Correlation)

\begin{tabular}{|c|c|c|c|}
\hline & $\begin{array}{c}\text { Open \& } \\
\text { Closed }\end{array}$ & Open & Closed \\
\hline \multicolumn{4}{|c|}{ Base-to-peak Amplitude: } \\
\hline P300 & .70 & .53 & .74 \\
\hline N100 & .18 & .08 & .12 \\
\hline $\mathrm{N} 200$ & .48 & .36 & .29 \\
\hline \multicolumn{4}{|c|}{ Peak-to-peak Amplitude: } \\
\hline N100-P300 & .61 & .52 & .50 \\
\hline N200-P300 & .43 & .59 & .47 \\
\hline \multicolumn{4}{|l|}{ Latency: } \\
\hline P300 & .65 & .58 & .42 \\
\hline N100 & .05 & -.07 & .09 \\
\hline N200 & .30 & .24 & .20 \\
\hline \multicolumn{4}{|l|}{ Area: } \\
\hline P300 & .61 & .42 & .57 \\
\hline \multicolumn{4}{|c|}{$\begin{array}{l}\text { Between-session reliability presented as } \\
\text { an intraclass correlation ( } \mathrm{r}^{\prime} \text { ) for multiple } \\
\text { ERP components. Correlations included } \\
\text { both visual procedures (eyes-open and } \\
\text {-closed); eyes-open (open), and eyes-closed } \\
\text { (closed) procedures, respectively. These } \\
\text { were computed from two-factor ANOVA } \\
\text { (subject } \times \text { session) in each case. }\end{array}$} \\
\hline
\end{tabular}


N200-P300 components were less than 0.5 , but those of the P300 and N100-P300 amplitudes and the P300 latency were more than 0.5 (Table 1). The highest between-session reliability for the P300 was obtained with the amplitude $\left(r^{\prime}=0.70\right)$.

In the eyes-open condition, a significant agreement $\left(\mathrm{r}^{\prime}>0.5\right)$ was observed for the P300, N100-P300 and N200-P300 amplitudes and the P300 latency. The P300 and N100-P300 amplitudes were also significantly reliable in the eyes-closed condition. No significant reliability was observed for the N100 and N200 amplitudes or latencies (Table 1). The correlations for the ERP measures such as the P300 amplitude and area in the eyes-closed condition exceeded those obtained in the eyes-open condition. Values for components of the N200 such as the N200-P300 and N200 amplitudes and the P300 latency in the eyes-open condition were higher than those obtained in the eyes-closed condition. The highest between session reliability was obtained from the P300 amplitude in the eyes-closed procedures $\left(r^{\prime}=0.74\right)$.

Within-session reliability (r): Pearson correlation coefficient: There were several interesting differences with respect to the within-session reliability. Only the correlation for the N200-P300 amplitude was generally significant ( $\mathrm{r}>$ 0.5 ) between the eyes-open and eyesclosed procedures. However, the Bartlett's T-test showed statistical significance with the inter-condition for all components of the amplitude, the P300 latency and area and the N200 latency (Table 2). The within-session reliability for the N100 latency was very low and not statistically significant $(r=0.06)$ between the eyes-open
TABLE 2.

Within-session reliability $(r)$

(Pearson Correlation Coefficient)

\begin{tabular}{cccc}
\hline & $\begin{array}{c}\text { Open } \times \\
\text { Closed }\end{array}$ & Open & Closed \\
\hline Base-to-peak Amplitude: & & & \\
P300 & $.43^{* * *}$ & $.45^{* * *}$ & $.58^{* * *}$ \\
N100 & $.23^{* *}$ & $.31^{* *}$ & -.18 \\
N200 & $.33^{* * *}$ & $.41^{* *}$ & .18 \\
Peak-to-peak Amplitude: & & & \\
N100-P300 & & & \\
N200-P300 & $.43^{* * *}$ & $.41^{* *}$ & $.39^{* *}$ \\
Latency: & $.51^{* * *}$ & $.62^{* * *}$ & $.37^{* * *}$ \\
P300 & & & \\
N100 & & & \\
N200 & $.25^{* *}$ & $.45^{* *}$ & $.37^{* *}$ \\
& .06 & .07 & .08 \\
& $.27^{* *}$ & .14 & $.38^{* *}$
\end{tabular}

Area:

P300

$.35 * * * \quad .39 * * \quad .47 * * *$

$* \mathrm{P}<0.05, * * \mathrm{P}<0.01, * * * \mathrm{P}<0.001$, (Bartlett's T- test)

Within-session reliability calculated as a Pearson coefficient correlation (r) for multiple ERP measures. Correlations were tested between the eyes-open and -closed conditions (open $\times$ closed), between the first and second block in the eyes-open (open) and eyes-closed (closed) conditions, respectively. Statistical significance (P) as calculated by Bartlett's T-test is indicated by asterisks (*, ** and ***) for each correlation. The values for P300 base-to-peak and N200-P300 peak-topeak amplitudes were highly significant in all cases $(\mathrm{P}<0.001)$.

and eyes-closed procedures.

The within-session reliability of the ERP measures was computed separately for each visual condition as the correlation between the first and second blocks (interblock). Table 2 shows the median of the reliability indices obtained in the eyesopen or eyes-closed condition. The P300 
amplitude obtained with the eyes closed and the N200-P300 amplitude obtained with the eyes open revealed correlation coefficients $>0.5$. The effect of the visual condition on the reliability is shown in Table 2. The correlations for the P300 amplitude, the P300 area and the N200 latency obtained with the eyes open were smaller than those with the eyes closed. However, the correlations for the P300 latency, the N200-P300 amplitude, N100 amplitude and N200 amplitude were larger with the eyes open than with the eyes closed. The inter-block reliability (withinsession reliability) was not significant (Bartlett's T-test) for the N100 and N200 amplitudes with the eyes closed, the N100 latency in both visual conditions, and the N200 latency with the eyes open.

The highest correlation was obtained for the N200-P300 amplitude with the eyes open $(\mathrm{r}=0.62, \mathrm{P}<.0001)$.

Habituation and dishabituation: Although the mean values of the P300 amplitude, P300 area and N200-P300 amplitude throughout the 8 sessions tended to be reduced in session 4 , no significant differences were observed (Fig. 1). The mean N100-P300 amplitude was essentially stable. The amplitudes of the N100 and N200 did not differ significantly over the 8 sessions. There were no statistically significant changes in the P300 latency. However a statistically significant effect in all 8 sessions was observed in the N100 $(\mathrm{F}[7,310]=2.28, \mathrm{P}=0.028)$ and the $\mathrm{N} 200$ latencies $(\mathrm{F}[7,310]]=3.21, \mathrm{P}=0.003)$ for both visual procedures. The N200 latency was markedly prolonged in sessions 4 and 5 as compared with sessions 1 and 2 . The values approached the initial values at sessions 7 and 8 . With the eyes-closed procedure, only the main session effect on

\section{A TRANSITION OF AMPLITUDE}

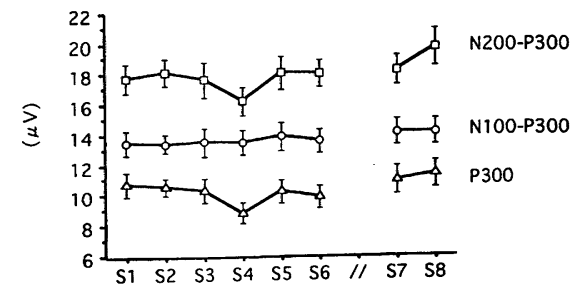

B TRANSITION OF N2OO LATENCY

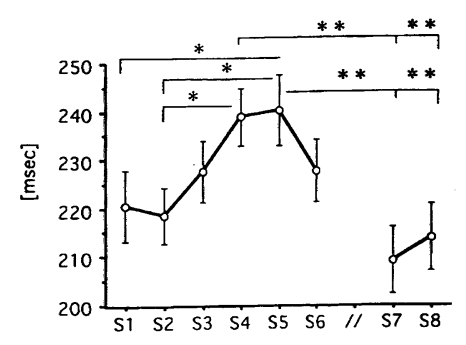

Fig. 1. Series of difference waveforms. N200-P300 peak-to-peak amplitude (top), N100P300 peak-to-peak amplitude (middle) and P300 base-to-peak amplitude (low) in A and the N200 latency in B are shown as a function of the session. The inter-session interval was 7-10 days except for the interval between 6 and 7 (one month), indicated by //. The vertical bars represent means \pm S.E. of the mean. Note that the N200 was clearly prolonged in latency from the initial sessions 1 and 2 to sessions 4 and 5 . The statistical significance of the differences were evaluated by Fisher's protected $\operatorname{LSD}(*: \mathrm{P}<0.05, * *: \mathrm{P}<0.01)$.

the N200 latency was statistically significant $(\mathrm{F}[7,150]=3.553, \mathrm{P}=0.0017)$ (Fig. 1B). No significant difference in the $\mathrm{N} 200$ latency $\quad(\mathrm{F}[7,150]=1.86, \quad \mathrm{P}=0.081) \quad$ was observed with the eyes open.

Age-dependent changes: The P300 latency is considered to vary with age (Polish, 1991). In the waveforms obtained, 
the P300 latency increased about $1.2 \mathrm{~ms}$ per year. The calculated P300 latency by age was observed to have a difference of plus $1.2 \mathrm{~ms} / \mathrm{year}$ over the age of 40 , and minus $1.2 \mathrm{~ms} /$ year under the age of 40 . When the P300 latency was recalculated by considering ages, there was no significant difference over the 8 sessions.

\section{Task correctness}

There were no significant differences among the 8 sessions as to the correctness of task performance (count and button tasks). All sessions had a correctness of over $95 \%$ for both tasks. There was no significant relationship between the ERP components and correctness.

\section{ERPs of frequent stimuli}

The P300 observed in frequent waveforms were not identified or classified as the traditional P300. However, for subsequent analysis, the P300-like values were measured using the same method as used for the P300 measures of rare waveforms.

Between-session reliability $\left(r^{\prime}\right)$ : intraclass correlation: The intraclass correlation coefficients $\left(\mathrm{r}^{\prime}\right)$ of the P300 amplitude, area and latency were 0.07, 0.29 and 0.45 , respectively.

Within-session reliability ( $r$ ): Pearson correlation coefficient: The within-session reliability (inter-condition) was 0.11 for the P300 amplitude, 0.099 for the area and 0.23 for the latency. The values for the P300 amplitude were larger in the inter-block with the eyes closed than with the eyes open, but the correlation for the P300 latency was higher with the eyes open.

Habituation and dishabituation: The amplitude of the P300 did not vary significantly among the initial 6 sessions, but it increased in sessions 7 and 8 . The
P300 latency and area did not change significantly across the 8 sessions. In each visual condition, no significant difference was observed in the other ERP components. A tendency toward a decrease in the P300 area through sessions 1 to 6 with an increase in session 8 was found with the eye-closed procedure $(\mathrm{P}=0.0527)$.

\section{Comparison of three waveforms}

The N100 amplitude was markedly reduced by subtraction, being decreased to about $30 \%$ of its original value (Fig. $2 \mathrm{~A}$ ). The N200 amplitude of the difference waveforms was significantly enhanced, with the mean value being increased to about $300 \%$ of that obtained in the original waveform (rare waveform). However, the P300 amplitude obtained from the difference waveforms was essentially unchanged from the original waveform. Except for session 5, there were no significant differences between the 8 sessions. Fig. 2 shows the average of three waveforms: the original waveform elicited by rare trials, the original waveform in frequent trials, and the difference waveform. The amplitude and area of the rare P300 waveforms were clearly decreased between the initial sessions ( 1 and 2) and session 5, and were restored in the subsequent sessions (Kinoshita et al., unpublished observation). However the values of the difference waveforms were less affected. The mean values of the P300 amplitude and area obtained by the subtracted waveforms in session 5 were larger than those obtained from the waveforms for rare stimuli (Fig. 2B). The P300 amplitude of the difference waveforms significantly exceeded that of the rare waveforms $(\mathrm{F}[1,78]=6.09, \mathrm{P}=0.016)$. 


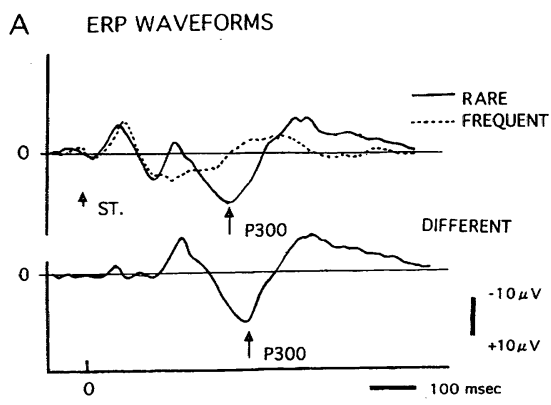

B TRANSITION OF P300 AMPLITUDE

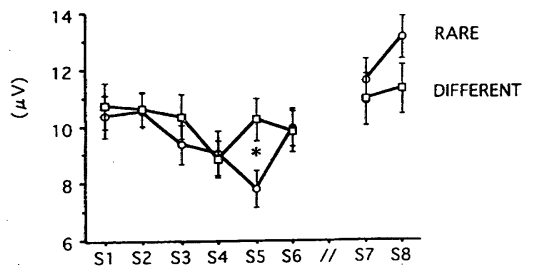

Fig. 2. Comparison of the original waveforms and the difference waveforms. Shown are three averaged waveforms: the waveforms caused by rare stimuli, the waveforms caused by frequent stimuli, and the difference waveforms at session 1 (A). Note that the N100 component was markedly canceled by subtraction, whereas the N200 component was clearly enhanced. The P300 latency seemed to be prolonged; however, there was no significant difference between the rare and the difference waveforms. Stimulus onset (ST. $\uparrow$ ) and P300 $(\uparrow)$ are shown in A. The transition of the P300 base-to-peak amplitude obtained from the rare waveforms and the difference waveforms is shown in B. A significant difference was observed at session 5 , as indicated by an asterisk $(\mathrm{P}=0.016)$.

\section{Discussion}

\section{Reliability}

In repeated studies of groups, the P300 amplitude was significantly stable in both the between-session (intraclass correlation; $\mathrm{r}^{\prime}=0.70$ ) as a trait marker and the within-session (Pearson coefficient; $r=$ 0.43 ) as a state marker. Furthermore, values of $r=0.45$ were found for the between blocks with the eyes open and $\mathrm{r}=0.58$ for the between blocks with the eyes closed as a state marker. Concerning the amplitude of the multiple P300 components, the intraclass correlation for the P300 amplitude $\left(\mathrm{r}^{\prime}=0.70\right)$ and the within-session reliability for the N200P300 amplitude ( $r=0.62$ : between blocks with the eyes open) were the most stable markers in each case, and were generally acceptable as measures of ERPs in group research because the values were more than 0.5 (Helmstadter, 1964). The present intraclass correlation for the P300 amplitude across 8 sessions $\left(r^{\prime}=0.70\right)$ was larger than that reported by Segalowitz and Barnes (1993, $\left.\mathrm{r}^{\prime}=0.61\right)$. The present withinsession reliability for the P300 amplitude ( $r=0.43$ for the inter-condition, $r=0.45$ for inter-block with the eyes open, $r=0.58$ for inter-block with the eyes closed) was comparable to findings in previous reports (Fabiani et al. 1987: reliability of probability effect by split-half method, $r=0.61$ at Pz; and Segalowitz and Barnes, 1993: reliability of rare waveforms at $\mathrm{Cz}, \mathrm{r}=0.48$ ). However, differences in recording sites or the number of rare tones existed among those studies. It was evident that the present within-session reliability for the amplitudes obtained from the difference waveforms were lower than those obtained from rare waveforms (Kinoshita et al., unpublished observation) as previously reported (Fabiani et al. 1987). This indicates that the present experimental method was correct. Thus the observed reliabilities adequately reflected the P300 
amplitude. In the present study, the P300 area that was obtained by integration of the positive elements between the time window (250-500 ms), was also examined by intraclass correlations and Pearson correlation coefficients. The intraclass correlation and the correlations between the two visual conditions were also comparable to those reported by Fabiani et al. (1987). The between-session reliability and within-session reliability for the P300 latency were generally lower than those for the P300 amplitude in the present study, as well as in previous studies (Sklare and Lynn, 1984; Fabiani et al. 1987; Segalowitz and Barnes, 1993). The present between-session reliability was higher than that reported by Fabiani et al. $(r=0.48)$. The within-session reliability was comparable to that reported by Polich $(\mathrm{r}=-0.32)$. The present low values for the between-session reliability for the P300 latency may be due to repetition of the trials 8 times, in addition to the effect of the number of rare tones, the recording site, or the method of split-half analysis. Indeed, the reliability was high for the initial two or three sessions. Although the correlations for the P300 latency as the within-session reliability may be different from that which is generally recommended $(\mathrm{r}<0.5)$, the coefficients were statistically significant (Bartlett's T-test) except for the inter-condition value. The low reliabilities for the P300 latency between the eyes-open and eyes-closed procedures and between the first and second blocks with the eyes closed, may be due to a visual influence on information processing and/or a contamination of the alpha wave by measurement error.

The N100-P300 amplitude is reportedly a stable marker, while the N100 amplitude is unstable (Segalowitz and Barnes, 1993). The present results were similar in that the N100-P300 amplitude was more stable across the 8 sessions than was the N100 amplitude. The low reliability for the N100 amplitude indicates that signal recognition is less affected by changes in subject than in signal detection (Parasuraman et al. 1982). The between-session reliability and the within-session reliability (range 0.06-0.08) for the N100 latency were very low. It was noted that the present subtraction procedure actually canceled the N100 component (see Fig. 2A). Thus, N100 measures may be difficult and unsuitable to examine in detail, because of the inclusion of measurement errors.

In the N200 components, the agreement as determined by the intraclass correlation, was generally higher than that for the N100 components. However, these values were lower than those for the P300 amplitude. The intraclass correlation for the N200-P300 amplitude $\left(r^{\prime}=0.43\right)$ was similar to that for the N200 amplitude $\left(r^{\prime}=0.48\right)$, since both amplitudes had less agreement than the P300 amplitude, area and latency. Concerning the within-session reliability, the inter-condition reliability for the N200-P300 amplitude $(r=0.51)$ was the highest of all the ERP measures. The within-session reliabilities for both the N200-P300 amplitude and the N200 amplitude were statistically significant except that for the N200 amplitude in the eyes-closed condition, which was similar to the finding for the N100 components. The lower reliability for the N200 amplitude than for the P300 indicates that signal discrimination may be more affected than signal recognition especially in the eyes-closed procedure (Hillyard and Kutas, 1983). 
The between-session reliability $\left(\mathrm{r}^{\prime}=\right.$ 0.30 ) and the within-session reliability (r; 0.14-0.38) for the N200 latency were low, but statistically significant except for that obtained with the eyes open. These results indicate that there was habituation of the N200 latency with the eyes-closed procedure (see below).

\section{Habituation and dishabituation}

The presently observed values for the P300 components such as P300 amplitude, area, latency, N100-P300 amplitude, N200P300 amplitude, N100 amplitude and N200 amplitude did not change significantly across the 8 sessions. These results indicate that the ERP components that were calculated from the difference waveforms may not develop habituation.

Of the difference waveforms, the N200 component was markedly enhanced. Thus analysis of this component may be useful, but the physiological meaning is unclear as Nd or MMN (see review by Hillyard and Kutas, 1983). Interestingly, the N200 latency in the eyes-closed condition displayed a type of significant habituation and dishabituation (Megela and Teyler, 1979; Lammers and Badia, 1989; Wesensten et al. 1990). It lengthened from sessions 1 and 2 to sessions 4 and 5, and was restored to the original duration at sessions 7 and 8 (see Fig. 1B). While a similar form of N200 latency was presented by Polich (1989), no details were provided. The N200 component probably connects discrimination between the target and nontarget tones; and the latency increased with the difficulty of the target presented (Ritter et al.1983; Michalaleski et al. 1986). According to the present evidence and previous reports, task discrimination may increase in difficulty as a function of repeated sessions. The amplitude varies widely depending on the complexity of the task and the arousal state of the subject (Segalowitz et al. 1990). In the present results, the mean value of the N200 amplitude tended to increase at session 6 , but there were no significant changes across the 8 sessions, suggesting that the N200 amplitude may not develop habituation. The N200 amplitude has been reported to increase during a drop in arousal level (Kevanishvili and Specht, 1978; Broughton, 1989). If the N200 amplitude increased in association with a decline in arousal in the opposite direction of the P300 amplitude, the significant prolongation of N200 latency may be due to a lowering of the arousal level of the subjects. However, this conclusion is tentative, because the increase in N200 amplitude was not significant, and similar results have been demonstrated with P300 habituation (Polich, 1991). The correctness of task performance was sustained and the EEG did not show a drowsy state, although the influence of changes in arousal level cannot be ignored as a nonspecific factor. It is possible that a slight drop in the arousal level occurs with habituation. According to the previous suggestion that the N200 component is more closely coupled to stimulus evaluation processes than is the P300 component and that it reflects the next phase of memory updating or decision closure (Ritter et al. 1979, 1983; Hillyard and Kutas, 1983), the time (session)-dependent prolongation of the N200 latency may be due more to a decrease in attention to the target tone than to a simple decline in arousal level. Familiarity with the stimulus and task performance (learning effect) may cause 
the subject to discriminate and evaluate the task automatically. It is important evidence that ERP measures, particularly obtained from the difference waveform may resist habituation and dishabituation which has been demonstrated in the original waveforms (rare trial; Kinoshita et al., unpublished observation). Further study is needed to clarify the mechanism underlying the habituation of the N200 latency, including a consideration of mismatch negativity (Näätänen, 1991).

\section{Physiological significance}

The P300 obtained by subtracting the waveforms induced by frequent stimuli from the waveforms caused by rare stimuli may reflect the probability effect (Fabiani et al. 1987), and may be stable for use in long-term repeated studies, because of an acceptable reliability and a resistance to habituation. It is important when recording ERPs repeatedly over long periods to use a stable, reliable method. The disappearance of habituation in the difference waveforms indicates that the waveform elicited by frequent stimuli may interact with those elicited by rare stimuli. No significant habituating phenomenon in any ERP measure was obtained from the frequent waveforms, and both the betweensession and within-session reliabilities were very low. However, the P300 amplitude had characteristics different from the traditional P300 and was reliably increased in sessions 7 and 8. A similar trend toward increases in P300 amplitude and area were observed with the difference waveforms. This phenomenon may be due to the influence of knowledge occurring by the end of the session (Lammers and Badia, 1989) or to the prolonged intervals between the tests.
It can be concluded that the P300 and N200-P300 amplitudes of the difference waveforms are in good agreement and are free of habituation over 8 sessions performed at 7-10 day intervals. These ERP measures are useful in repeated studies of groups as both trait and state makers, whether or not the difference waveform has any physiological significance.

\section{References}

Blackwood, D.H.R., Whalley, L.J., Christie, J.E., Blackburn, I.M., St Clair, D.M. et al. (1987). Changes in auditory P3 event-related potential in schizophrenia and depression. Br. J. Psychiatry 150, 154-160.

Broughton, R.J. (1989). Evoked potentials and sleep-wake states in man. In Sleep ' 88. Stuttgart, ed. Horne, J., pp.6-10. New York: Gustav Fisher Verlag.

Fabiani, M., Gratton, G., Karis, D. and Donchin, E. (1987). Definition, identification, and reliability of measurement of the P300 component of the event-related brain potential. Advances in Psychophysiology 2, 1-78.

Fitzgerald, P.G. and Picton, T.W. (1983). Eventrelated potentials during the discrimination of improbable stimuli. Biol. Psychiatry 17, 241-276.

Helmstadter, G.C. (1964). Principles of psychological measurement. New York: AppletonCentury-Crotts.

Hillyard, S.A. and Kutas, M. (1983). Electrophysiology of cognitive processing. Ann. Rev. Psychology 34, 33-61.

Kevanishvili, Z.S. and Specht, V. (1978). Human slow auditory evoked potentials during natural sleep and during induced sleep. Electroencephalogr. Clin. Neurophysiol. 47, 280-288.

Lammers, W.J. and BADIA, P. (1989). Habituation of P300 to target stimuli. Physiology and Behavior 45, 595-601.

Megela, A.L. and Teyler, T.J. (1979). Habituation and the human evoked potential. J. Comp. 
Physiol. Psychol. 93, 1154-1170.

Michalalewski, H., Prasher, D. and Starr, A. (1986). Latency variability and temporal interrelationships of the auditory eventrelated potentials (N1, P2, N2, and $\mathrm{P} 3$ ) in normal subjects. Electroencephalogr. Clin. Neurophysiol. 65, 59-71.

NäÄtänen, R. (1991). Mismatch negativity outside strong attentional focus: a commentary on Woldorff et al. Psychophysiology 28, 478484.

Parasuraman, R., Richer, F. and Beatty, J. (1982). Detection and recognition: concurrent processes in perception. Perception \& Psychophysics 31, 1-12.

Polich, J. (1989). Habituation of P300 from auditory stimuli. Psychobiology 17, 19-28.

PoLICH, J. (1991). P300 in clinical applications: Meaning, method, and measurement. Am. J. EEG Technol. 31, 201-231.

PRITCHARD, W.S. (1986). Cognitive event-related potential correlates of schizophrenia. Psychol. Bull. 100, 43-66.

RACZKowSKI, D. and KALAT, J.W. (1974). Reliability and validity of some handedness questionnaire items. Neuropsychologia 12, 43-47.

Ritter, W., Simson, R., Vaughan, H.G.Jr. and FRIEDMAN, D.A. (1979). A brain event related to the making of a sensory discrimination. Science 203, 1358-1361.

Ritter, W., Simson, R. and Vaughan, H.G.JR. (1983). Event-related potential correlates of two stages of information processing in physical and semantic discrimination tasks. Psychophysiology 120, 168-179.

Segalowitz, S.J., Ogilivie, R.D. and Simons, I.A. (1990). An ERP state measure of arousal based on behavioral criteria. In Sleep'90. Stuttgart, ed. Horne., J., pp.23-25. New York: Gustav Fischer Verlag.

SegalowttZ, S.J. and Barnes, K.L. (1993). The reliability of ERP components in the auditory oddball paradigm. Psychophysiology 30, 451459.

Shrout, P.E. and Fleiss, J.L. (1979). Intraclass correlations: Uses in assessing rater reliability. Psychol. Bull. 86, 420-428.

SkLARE, D.A. and LyNN, G.E. (1984). Latency of the P300 event-related potential: Normative aspects and within subject variability. Electroencephalogr. Clin. Neurophysiol. 11, 600-602.

Wesensten, N.J., Badia, P. and Harsh, J. (1990). Time of day, repeated testing, and interblock interval effects on P300 amplitude. Physiology and Behavior 47, 653-658. 